\title{
A transcriptomic reporter assay employing neutrophils to measure immunogenic activity of septic patients' plasma
}

Prasong Khaenam ${ }^{1,2}$, Darawan Rinchai ${ }^{2}$, Matthew C Altman', Laurent Chiche ${ }^{3}$, Surachat Buddhisa ${ }^{2}$, Chidchamai Kewcharoenwong ${ }^{2}$, Duangchan Suwannasaen ${ }^{2}$, Michael Mason' ${ }^{1}$, Elizabeth Whalen ${ }^{1}$, Scott Presnell', Wattanachai Susaengrat ${ }^{4}$, Kimberly O'Brien ${ }^{1}$, Quynh-Ahn Nguyen ${ }^{1}$, Vivian Gersuk', Peter S Linsley ${ }^{2}$, Ganjana Lertmemongkolchai ${ }^{2^{*}}$ and Damien Chaussabel ${ }^{1 *}$

\begin{abstract}
Background: There are diverse molecules present in blood plasma that regulate immune functions and also present a potential source of disease biomarkers and therapeutic targets. Genome-wide profiling has become a powerful method for assessing immune responses on a systems scale, but technologies that can measure the plasma proteome still face considerable challenges. An alternative approach to direct proteome assessment is to measure transcriptome responses in reporter cells exposed in vitro to plasma. In this report we describe such a "transcriptomic reporter assay" to assess plasma from patients with sepsis, which is a common and severe systemic infectious process for which physicians lack efficient diagnostic or prognostic markers.

Methods: Plasma samples collected from patients with culture-confirmed bacterial sepsis and uninfected healthy controls were used to stimulate three separate cell types - neutrophils, peripheral blood mononuclear cells, and monocyte-derived dendritic cells. Whole genome microarrays were generated from stimulated cells to assess transcriptional responses. Unsupervised analysis and enriched functional networks were evaluated for each cell type. Principal component analyses were used to assess variability in responses. A random K-nearest neighbor - feature selection algorithm was used to identify markers predictive of sepsis severity, which were then validated in an independent data set.
\end{abstract}

Results: Neutrophils demonstrated the most distinct response to plasma from septic patients with 709 genes showing altered expression profiles, many of which are involved in established immunologic pathways. The amplitude of the neutrophil transcriptomic response was shown to be correlated with sepsis severity in two independent sets of patients comprised of 64 total septic patients. A subset of 30 transcripts selected using one set of patients was demonstrated to have a high degree of accuracy (82-90\%) in predicting sepsis severity and outcomes in the other independent set. This subset included several genes previously established in sepsis pathogenesis as well as novel genes.

Conclusions: These results demonstrate both the suitability and potential clinical relevance of a neutrophil reporter assay for studying plasma, in this case from septic patients. The distinctive transcriptional signature we found could potentially help predict severity of disease and guide treatment. Our findings also shed new light on mechanisms of immune dysregulation in sepsis.

Keywords: Transcriptomic reporter assay, Sepsis, Plasma, Microarray, Systems immunology

\footnotetext{
* Correspondence: ganja_le@kku.ac.th; dchaussabel@benaroyaresearch.org

${ }^{2}$ Centre for Research and Development of Medical Diagnostic Laboratories

(CMDL), Faculty of Associated Medical Sciences, Khon Kaen University, 123

Friendship Highway, Muang, Khon Kaen 40002, Thailand

'Systems Immunology Division, Benaroya Research Institute, 1201 Ninth

Avenue, Seattle, WA 98101, USA

Full list of author information is available at the end of the article
} 


\section{Background}

The immune system plays a pivotal role in maintaining the balance between health and disease. Profiling immunological perturbation holds potential for elucidating the pathogenesis of a wide range of diseases. Currently available high-throughput profiling technologies and emerging systems immunology analysis approaches enable the study of clinical samples on a system-wide scale and provide unbiased tools for investigation of immune responses $[1,2]$. Whole blood transcriptome profiling has been employed to investigate a wide range of conditions [3-5]. Plasma, which is a valuable source of potential biomarkers, is an attractive alternative for profiling molecular changes associated with disease pathogenesis and progression on a systems scale. However, robust, costeffective and reproducible technologies needed for measuring plasma protein abundance on a systems scale are still lacking. Most prevalent is mass spectrometry, however this lacks well-established reference databases and is biased toward detecting high-concentration compounds, which are major limitations for assessment of the plasma proteome by this technology $[1,6]$.

So-called "transcriptomic reporter assays" provide an alternate means to assess perturbations in plasma on a system-wide scale [7]. This strategy consists of measuring whole genome transcriptional responses elicited in reporter cells exposed in vitro to patient plasma. This type of approach has already proven useful in studies of several immunologically mediated diseases. It was employed to help unravel the pathogenesis of systemic onset juvenile idiopathic arthritis, eventually leading to the adoption of a novel therapeutic modality for treatment of this disease $[7,8]$. It has been used to identify candidate biomarker signatures in patients prior to the clinical onset of type 1 diabetes mellitus [9]. It has contributed to identifying pathways of pancreatic islet cell destruction in islet cell transplantation $[10,11]$. Despite these and other successes, this approach has not yet been widely explored or adopted.

Sepsis is a clinical syndrome related to dysregulated systemic inflammation in response to an underlying infection. Uncontrolled production of cytokines and chemokines is believed to play a role in sepsis severity [12]. Early recognition leading to targeted antimicrobial and supportive therapy is critical to survival and each hour that treatment is delayed can markedly increase mortality [12-14]. However, due to an incomplete understanding of sepsis pathogenesis, criteria for rapid diagnosis and severity assessment are limited and based largely on non-specific clinical signs of systemic inflammation and organ dysfunction $[15,16]$. Several biomarkers have been studied in attempts to provide more simple, rapid, and accurate methods for diagnosis and prognosis of sepsis [17]. These include C-reactive protein, procalcitonin, triggering receptor expressed on myeloid cells 1 (TREM-1) and others [17-19]. While several small studies have shown correlation of such proteins to sepsis severity and outcomes, these proteins have not proven reliable on a larger scale and are not routinely used in clinical practice $[18,20]$. Recent studies suggest that combined use of multiple biomarkers may be more accurate, however at present these remain investigational [19].

Our study evaluated responses of three different cell types to stimulation with septic plasma: polymorphonuclear cells (PMNs), peripheral blood mononuclear cells (PBMCs), and monocyte-derived dendritic cells (MoDCs). PMNs and PBMCs were selected as these constitute the primary types of leukocytes in peripheral blood and are key in control of infections. MoDCs were selected because they are known to play a central role in the immune system and are able to respond to diverse immune signals. Each of these cell populations functioned as a so-called "reporter cell system" to investigate the transcriptional response to septic plasma. We demonstrate the utility of a reporter cell system for elucidating immune pathogenesis of a complex disease such as sepsis and the potential relevance of this approach for predicting prognosis in sepsis.

\section{Methods}

\section{Ethics statement}

The study was approved by the ethical review committees of Khon Kaen University and Khon Kaen Regional Hospital (Khon Kaen, Thailand) and the Institutional Review Board of Benaroya Research Institute (Seattle, WA). Participants provided written informed consent to participate in this study. Written informed consent was obtained from parents or guardians on behalf of the minor/child participant in this study.

\section{Plasma collection}

Septic patients were enrolled from Khon Kaen Regional Hospital, Khon Kaen, Thailand. Patients who met at least two of the criteria for severe inflammatory response syndrome (SIRS) were enrolled in the study $[3,15]$. As part of the routine investigations, clinical specimens were collected for bacterial culture within $24 \mathrm{~h}$ following SIRS diagnosis. Only blood samples obtained from patients who were retrospectively diagnosed with cultureproven sepsis were retained for further analyses. Patients with negative blood cultures were excluded. Severe sepsis was defined according to the current guidelines from the Surviving Sepsis Campaign [15]. These criteria include several clinical and laboratory findings of sepsisinduced tissue hypoperfusion or organ dysfunction. We used the subset of these criteria for which the necessary data had been collected for our patient cohort: elevation in creatinine to $>2.0 \mathrm{mg} / \mathrm{dl}$, elevation in bilirubin to $>$ $2.0 \mathrm{mg} / \mathrm{dl}$, platelet count $<100,000 / \mu \mathrm{L}$, and sepsis 
induced hypotension (septic shock). In hospital death was also counted as severe sepsis. Demographic and clinical data were recorded for all subjects (Additional files 1,2 , and 3). Uninfected healthy controls were selected as individuals who had no signs of acute infectious diseases during the previous 3 months or at the time of the study. Uninfected controls also had to have normal blood counts, normal fasting blood glucose, and normal glycosylated hemoglobin. Three milliliters of whole blood was collected from each patient and healthy control into heparinized tubes (BD Biosciences). For sepsis patients, samples were grouped as drawn either in the first $48 \mathrm{~h}$ of admission, or at $>48 \mathrm{~h}$ after admission. To separate plasma, blood samples were centrifuged at 2,000 rpm for 10 minutes and the plasma component was transferred into a cryogenic vial and stored at $-80^{\circ} \mathrm{C}$ until used.

\section{PMNs and PBMCs isolations from healthy volunteers}

Blood samples from three additional healthy volunteers were used in subsequent cell isolation procedures. PMNs were isolated from heparinized venous blood by $3.0 \%$ dextran T-500 sedimentation and Ficoll-Paque PLUS centrifugation (Amersham Biosciences) as previously described [21]. The purity of isolated cells was generally more than $95 \%$ as determined by flow cytometry (FACSCalibur, Becton Dickinson) [22]. PBMCs were isolated from whole blood samples by centrifugation through a Ficoll-Paque Plus (Sigma Aldrich) density gradient.

\section{Generation of MoDCs}

A portion of the isolated PBMCs was subsequently used for MoDCs generation as previously described [22,23]. MoDCs were harvested and resuspended in serum-free RPMI-1640 medium (Gibco), $5 \times 10^{5}$ cells/well were plated into a 24-well tissue culture plate (Corning) for $24 \mathrm{~h}$. The resulting cells were determined to be $>95 \%$ CD $11 c^{+}$by flow cytometry.

\section{Cell culture}

Cell cultures were performed as described by Pascual et al. [7]. Two million PMNs or one million PBMCs were resuspended in serum-free RPMI-1640 medium (Gibco) and added to either $5 \mathrm{ml}$ or $2 \mathrm{ml}$ culture tubes (Becton Dickinson), respectively. Five hundred thousand MoDCs were seeded into 24 well tissue culture plates (Corning) at $1 \times$ $10^{6}$ cells $/ \mathrm{ml}$ and rested for $24 \mathrm{~h}$ before the experiments. Cells were cultured with medium alone or a plasma sample in a final concentration of $20 \%$. After $6 \mathrm{~h}$ incubation at $37^{\circ} \mathrm{C}$ in $5 \% \mathrm{CO}_{2}$, cells were harvested, washed twice with phosphate buffered saline, homogenized in RLT buffer (RNeasy mini kit; QIAGEN), and stored at $-80^{\circ} \mathrm{C}$ until use.

\section{RNA preparation and microarray}

Total RNA was isolated using the RNeasy Mini kit (QIAGEN) according to the manufacturer's instructions. RNA integrity number (RIN) was determined by using an Agilent 2100 Bioanalyzer (Agilent). Qualified samples (RIN $>6$ or presence of $28 \mathrm{~s}$ and $18 \mathrm{~s}$ rRNA) were retained for further processing. Total RNA was amplified and labeled using the Illumina TotalPrep RNA Amplification Kit (Ambion). Labeled cRNA was hybridized overnight to Human HT-12 V4 BeadChip array (IIlumina), washed, blocked, stained and scanned on an Illumina HiScan instrument following the manufacturer's protocols.

\section{Data acquisition and background subtraction}

GenomeStudio was used to generate signal intensity values from the scans and perform background subtraction. Post-hybridization quality controls were done by the standard metrics provided by the manufacturer. Data from each cell type and each culture experiment were processed independently. All possible outliers were excluded from the expression data set by metrics for posthybridization quality controls.

\section{Data normalization}

All data analyses were performed using $\mathrm{R}$ (version 2.14.0; http://cran.r-project.org/bin/windows/base/old/2.14.0/). Data pre-processing of background subtracted data was performed by using the preprocessCore package from Bioconductor. Pre-processing included rescaling intensity by quantile normalization. After normalization, expressions were floored with intensities $<10$ set to 10 . Transcripts with detection p-value of less than or equal to 0.01 in at least one sample (PALO) were selected for further analysis. Samples from the same cell type and batch were normalized to the average intensity of samples cultured in medium alone. A filter was set to include only transcripts that had at least two-fold changes and 100 intensity differences compared to medium control. Background subtracted and processed data from these experiments have been deposited at NCBI's Gene Expression Omnibus database (http://www.ncbi.nlm.nih.gov/geo/), with accession numbers GSE49758. To facilitate data sharing and interactive data analysis, we created a data portal (https://gxb. benaroyaresearch.org/tra/tra-paper/tra-landing.gsp) to store and analyze background subtracted data from all three experiments (see [24] for tutorial).

\section{Unsupervised analysis}

Principle component analysis (PCA) was performed using the $\mathrm{R}$ function "prcomp". The first two principal components, PC1 and PC2, were plotted against each other. Each colored dot represents an individual sample. Euclidean distances were calculated by measuring the distance from each sample to the average of samples 
stimulated with uninfected plasma. The comparison between severe and non-severe sepsis was performed using the Mann-Whitney $U$-test. Hierarchical clustering analysis was performed using the function "heatmap.2" from the R package "gplots". Euclidean distance and complete linkage methods were used by default.

\section{Feature selection}

Transcripts that were differentially expressed between study groups were selected by Random k-Nearest Neighbor - Feature Selection (RKNN-FS) using the R package "rknn" [25]. A RKNN classifier consists of an ensemble of base k-nearest neighbor models (number of neighbors $=5$ ), each constructed from a random subset of the input variables. Features were selected by ranking the importance of the PALO transcripts.

\section{Pathway analysis}

Gene ontology analyses were performed using GeneGo MetaCore pathway analysis tool (Thomson Reuters, NY). The default background gene list was used for all enrichment analyses including process networks, pathway map folders, and pathway maps. Pathway maps are the collection of pathways grouped into folders according to main cell processes, protein functions, and diseases. Map Folders are the collection of pathways grouped into folders according to main biological processes. Statistical significance was ascertained by using a threshold of false discover rate $(F D R)<0.05$. The network builder tool using the shortest possible path with no more than 2 steps was used to represent functional interactions. Upstream transcription factors were identified for lists of over-expressed genes using the Transcription Factors tool.

\section{Class prediction}

To determine the performance of our predictive signature, class prediction of binary variables was carried out by support vector machine (SVM; package "e1071") and random forest (RF; package "randomForest") algorithms. These machine learning methods are robust, well-accepted and commonly used methods for class prediction. Receiver operating characteristic (ROC) curves were constructed using the R package "ROCR". Area under the curve (AUC) and confidence intervals were calculated using the R package "Hmisc".

\section{Results}

Septic plasma elicits transcriptional responses that can be measured on a systems scale in a cell reporter assay

We first set out to determine which cell reporter system would be most amenable to detect meaningful changes in response to septic plasma in vitro. PMNs, PBMCs and monocytes were isolated from two healthy donors
(Table 1). Monocytes were cultured with interleukin 4 (IL4) and granulocyte-macrophage colony-stimulating factor (GM-CSF) to generate MoDCs. Plasma samples were obtained from a first set of patients with cultureconfirmed sepsis $(n=12)$ and from uninfected healthy controls $(n=6)$. Cells were cultured for 6 hours in medium alone (unstimulated) or in the presence of plasma (stimulated) using a final concentration of 20\% (Figure 1). Microarray data were generated to assess transcriptional responses on a genome-wide scale. PALO filtering returned 21,236, 25,728, and 23,589 transcripts in PMNs, PBMCs, and MoDCs respectively. Transcripts were selected that changed by at least 2-fold and 100 intensity differences (2FC100DIF) in response to plasma stimulation compared to unstimulated cells. To reduce data dimensionality and facilitate the comparison among cell types, principal component analyses (PCA) were carried out. PCA plots of data obtained from the 3 different reporter cell systems (PMNs, PBMCs, and MoDCs) show changes in transcription that can be attributed to responses to plasma stimulation (Figure 2). The best separation between sepsis and uninfected plasma samples was observed in PMNs (Figure 2, top left panel). This result was confirmed by calculating the Euclidean distance from the center of uninfected controls (Additional file 4). These results demonstrate the feasibility of a "reverse proteomics" approach to detect the presence of immunomodulatory factors in the blood of sepsis patients. While responses measured in PMNs and PBMCs were consistent for both cell donors (Figure 2, upper and middle right panels), significant donor-to-donor variation was observed in MoDCs (Figure 2, lower right panel).

PMNs mount a robust immune transcriptional program in response to septic plasma

To test how our approach could best be used for monitoring immunomodulatory factors in the blood of sepsis patients, we next compared the transcriptional programs elicited by septic plasma in PMNs, PBMCs, and MoDCs. Septic plasma samples $(n=6 / 24)$ that were found to induce the most robust responses consistently across the three cell reporter systems were selected (Figure 2, Additional file 5). Transcripts changing by at least 2 -fold

\section{Table 1 Overview of experimental design and sample} sizes

\begin{tabular}{ccc}
\hline Experiments & Cell donors & Plasma sources \\
\hline I & Healthy subjects $(n=2)$ & Uninfected subjects $(n=6)$ \\
& & Sepsis subjects $(n=12)$ \\
II & Healthy subjects $(n=1)$ & Uninfected subjects $(n=18)$ \\
& & Sepsis subjects $(n=29)$ \\
III & Healthy subjects $(n=2)$ & Uninfected subjects $(n=19)$ \\
& & Sepsis subjects $(n=35)$ \\
\hline
\end{tabular}




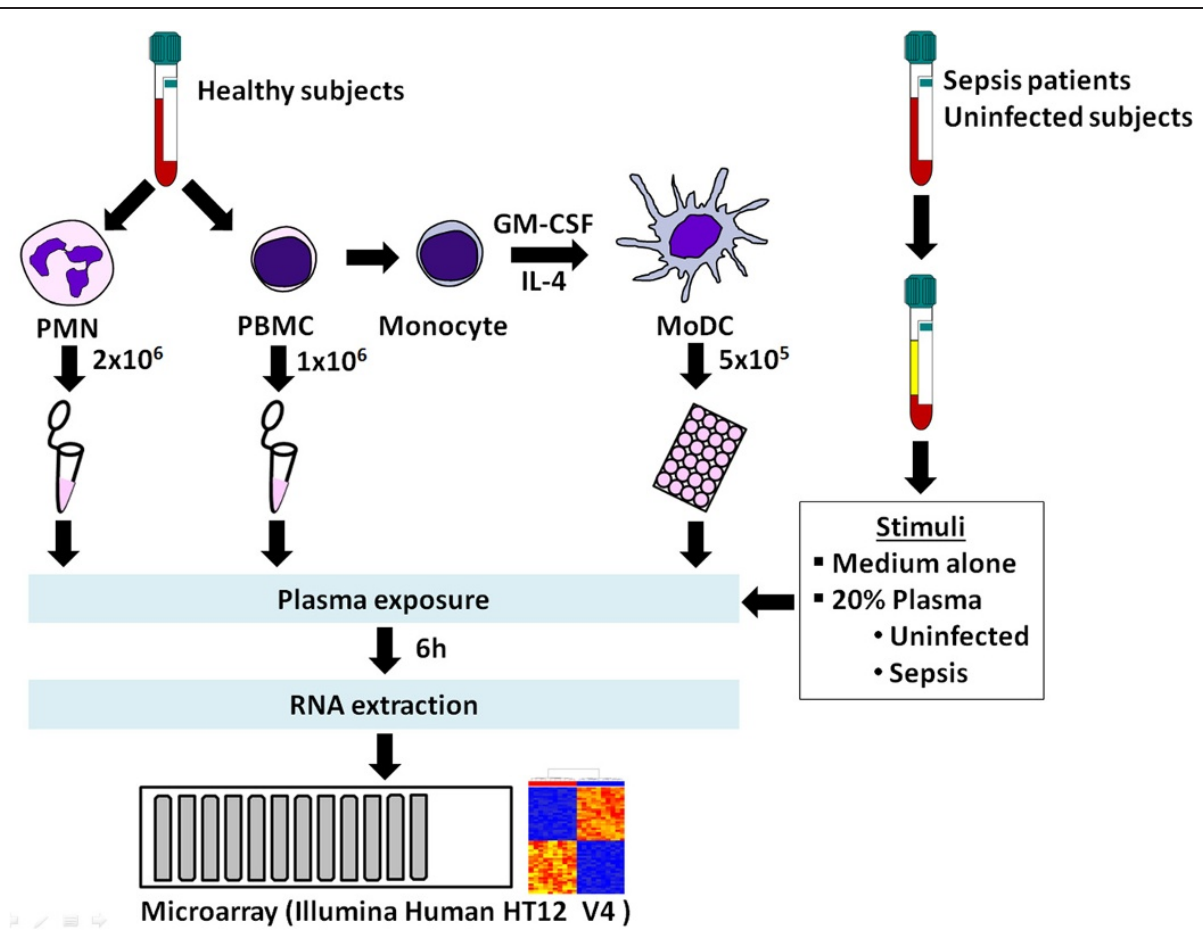

Figure 1 Schematic illustration of the experimental design and procedures. Experiments were performed using 3 independent sets of patient samples. Cells were isolated from healthy volunteers and the responses to plasma measured after $6 \mathrm{~h}$ of culture using whole genome Illumina Human HT-12 V4 BeadChips. Plasma samples were obtained from patients with culture-confirmed sepsis $(n=12,29$, and 35 in experiments I, II and III, respectively) and control subjects with no infection ( $n=6,20$, and 20 in experiments I, II and III, respectively). In experiment I, three types of leukocyte populations were isolated from two healthy volunteers: PMNs, PBMCs and monocytes. In experiments II and III, only PMNs were used.

compared to their own unstimulated controls in each reporter system (709, 366 and 452 transcripts for PMNs, PBMCs and MoDCs, respectively) were combined to generate a list of 1,366 transcripts (Additional files 6 and 7). The number of transcripts passing this 2-fold cut off was the highest in PMNs and the majority of these transcripts are over-expressed (Table 2). In contrast, there were fewer changes recorded in PBMCs and MoDCs and for the majority of those genes, transcript abundance decreased upon exposure to septic plasma.

In an effort to determine potential molecules present in septic plasma inducing these patterns of gene expression, we selected those genes over-expressed in each of the 3 reporter cell types and predicted the upstream transcription factors using GeneGo MetaCore. One hundred and sixty-one transcription factors were identified in total (Additional file 8). There was significant overlap among the 3 lists of transcription factors with 27 predicted for all 3 cell types, including several well established in core immune pathways such as STAT3, STAT4, STAT5A, STAT5B, and CREB1. These findings are consistent with the idea that a relatively small number of molecules are driving the observed transcriptional responses and could suggest future studies to determine the relevant immunomodulatory molecules in septic plasma.

To better understand the observed gene expression patterns and the functions of those genes, a heatmap was generated with the 1,366 transcripts differentially expressed in at least one cell type (Figure 3 ). We performed hierarchical clustering and further characterized the function of transcripts in each of the resulting clusters by using the GeneGo MetaCore pathway analysis tool. Networks with statistically significant enrichment of functional annotations were found in 5 out of 12 clusters. Most of the enriched functional networks are relevant to immune processes. Cluster $1(n=267)$, the largest cluster, is formed by transcripts over-expressed in PMNs. This cluster contains many genes involved in neutrophil function (e.g. CD177, IL1R2, NLRP3, TNFSF8, FCGR1A, FCGR1B, and FCGR1C). Cluster $3(\mathrm{n}=84)$ contains transcripts over-expressed in PBMCs, MoDCs and to a lesser extent PMNs. This cluster contains genes enriched for cell cycle and apoptotic mechanisms (e.g. CDKN1A, GADD45A, GAA45G, SMAD7, etc). Cluster 5 ( $\mathrm{n}=39$ ) is over-expressed in PMNs but under-expressed in PBMCs. This is a smaller cluster but it contains several important immune-related transcripts (e.g. CCL2, 

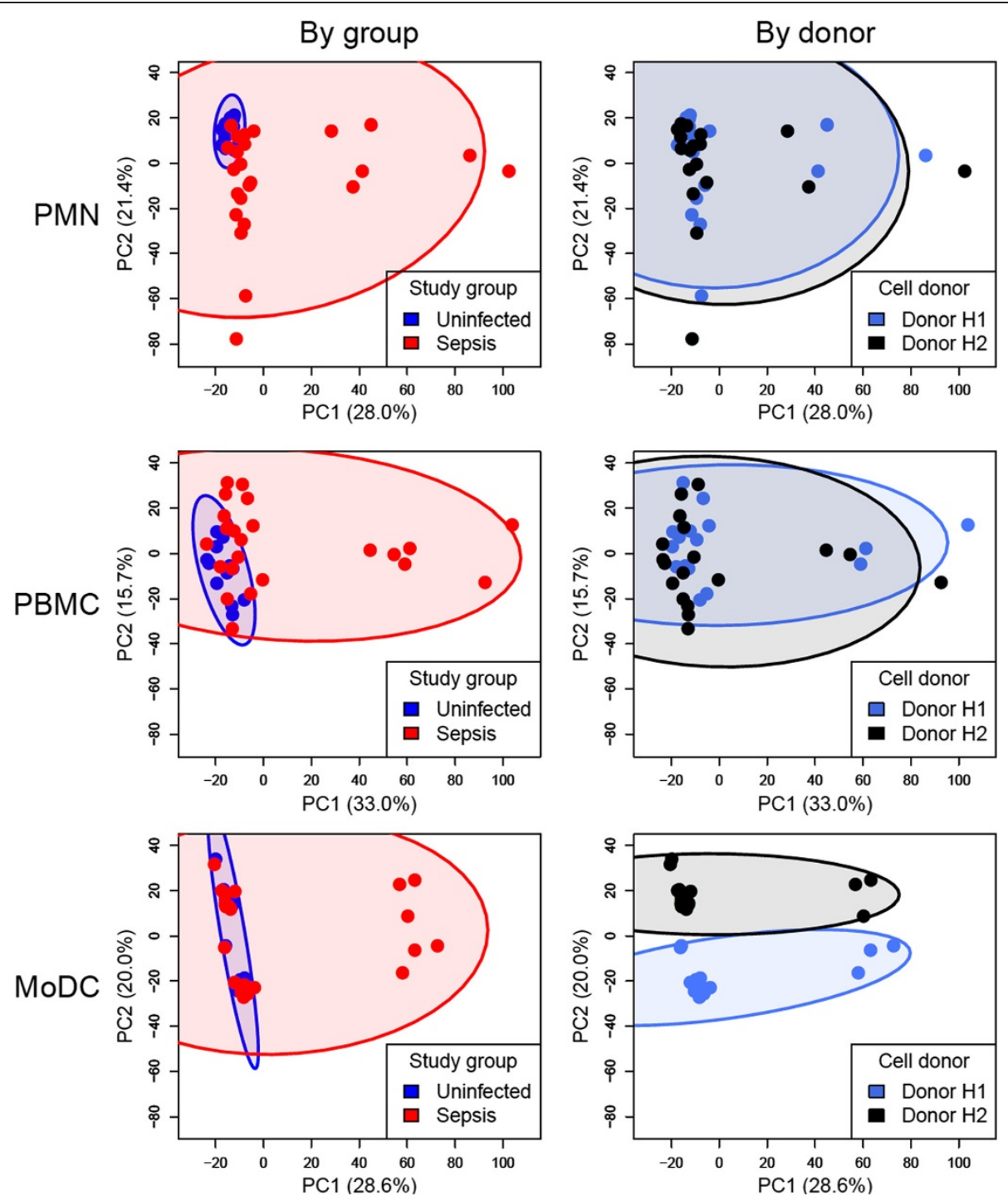

Figure 2 Principal component analyses of transcriptional responses to septic plasma in three different reporter cells systems. The transcriptional response to septic plasma measured by microarrays was analyzed separately for each reporter cell system. Fold-changes were calculated by normalizing the expression levels of cell cultures stimulated with plasma to their respective unstimulated cell cultures. Transcripts passing a filter criteria of 2 fold-change and 100 difference in intensity (2FC100DIF) were used for principal component analysis (PCA). Scores from principal component 1 (PC1) and principal component 2 (PC2), which explain approximately 50\% of the variability, were plotted. Samples were color-coded according to study groups (blue = uninfected plasma; red = septic plasma; left panels) or cell donors (blue = donor $\mathrm{H} 1$; black = donor $\mathrm{H} 2$; right panels). Each ellipse indicates the $95 \%$ confidence interval of data from the corresponding group (indicated by colors). Numbers in parentheses indicate percentage of variance.

Table 2 Number of over-expressed and under-expressed transcripts induced by septic plasma

\begin{tabular}{ccc}
\hline $\begin{array}{c}\text { Cell } \\
\text { types }\end{array}$ & $\begin{array}{c}\text { Number of over-expressed } \\
\text { transcripts }\end{array}$ & $\begin{array}{c}\text { Number of under- } \\
\text { expressed transcripts }\end{array}$ \\
\hline PMNs & 465 & 358 \\
PBMCs & 168 & 442 \\
DCs & 125 & 488 \\
\hline
\end{tabular}

CCL20, CXCL2, IL1RA, IL1A, IRAK3, and TLR2). Innate immune pathways and chemotaxis are the major functions of the genes in this cluster; specific induction of these genes in PMNs is consistent with their known role as the immune cells that respond immediately to bacterial infection. Cluster $6(\mathrm{n}=135)$ is under-expressed in PBMCs and is enriched in immune mediators involved in chemotaxis (e.g. CCL3, CCL3L1, CXCL5, and CXCL16), IFN-gamma signaling (e.g. CASP5, FCAR and $C F B$ ), and proliferation and morphogenesis (e.g. MMP19 and NRP1). Lastly, cluster 7 $(\mathrm{n}=178)$ is under-expressed in all reporter systems. The 


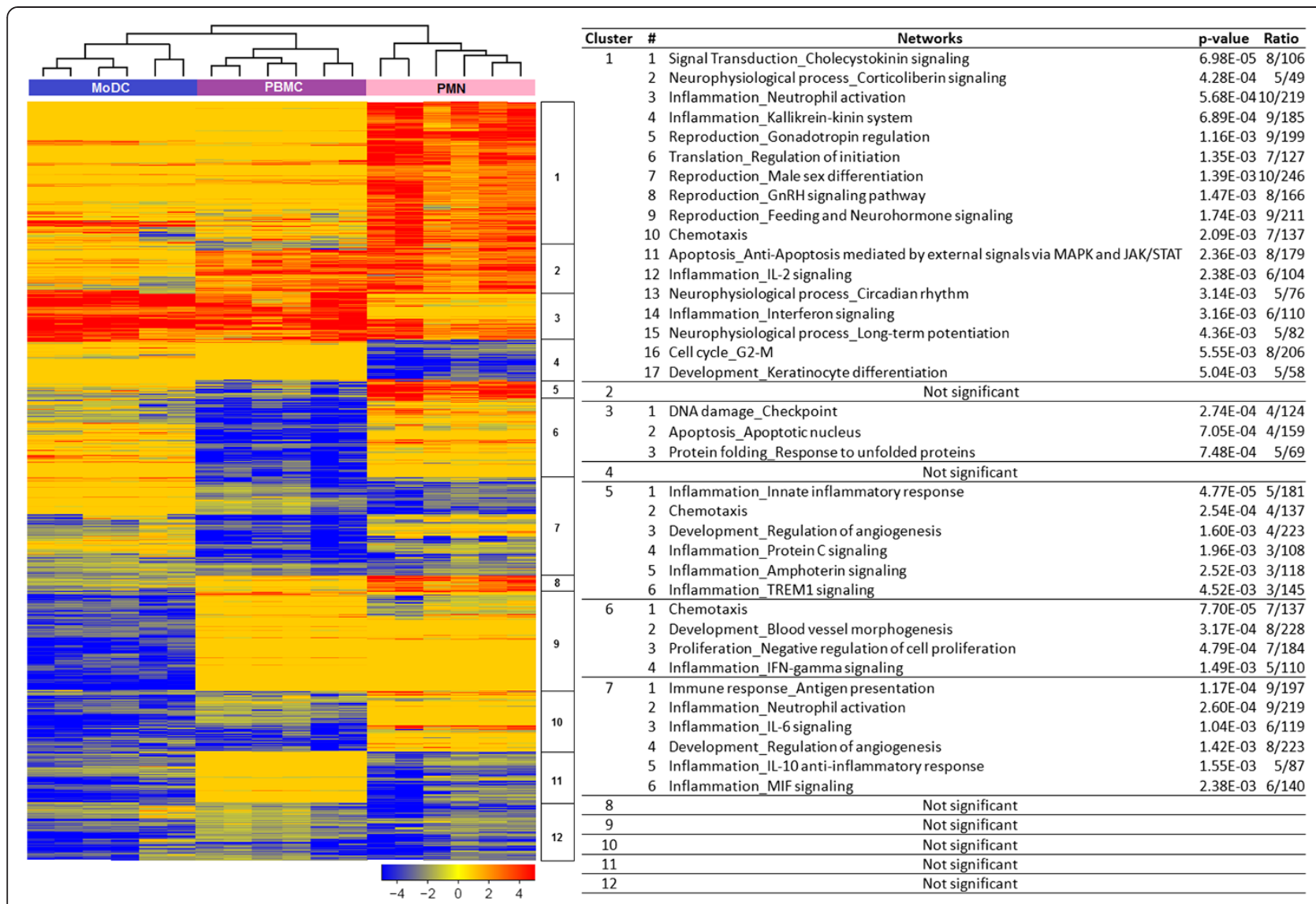

Figure 3 Transcriptional programs elicited by septic plasma in three reporter cell systems. Six samples, which provided the highest and most consistent response in all three reporter cell systems were selected (Additional file 5). Transcripts passing a filter criterion of 2 fold-change compared to medium controls were used $(n=1,366)$. Transcripts were organized by hierarchical clustering (Euclidean distance) according to similarities in expression profiles. Each row represents a transcript and each column an individual sample. The heatmap shows fold-change compared to unstimulated cell cultures. Red indicates over-expressed and blue indicates under-expressed transcripts. Clusters are identified by a number on the right side of the heatmap. Each cluster was annotated using the GeneGo MetaCore pathway analysis tool. P-values indicate enrichment significance for the networks based on hypergeometric distribution. Ratios indicate the number of molecules in the query set over the total number of molecules in the network.

enriched functional networks for this cluster are relevant to both innate and adaptive immune responses, for example, antigen presentation (e.g. CD58 and STAT1), and neutrophil activation (IL6, NFKB2, and CXCL1). Taken together, these results support the use of PMNs as a useful reporter cell type for sensing immunomodulatory constituents of septic plasma in our reverse proteomic assay system.

\section{A wide range of responses to septic plasma samples is observed in the PMN reporter assay system}

Next we sought to further investigate factors that account for the heterogeneity of the responses observed in PMNs exposed to plasma from the initial cohort of septic patients. Plasma samples from a larger independent set of septic $(n=29)$ and uninfected $(n=18)$ subjects were tested on PMNs collected from one of the cell donors used in our first experiment. PALO filtering returned 21,374 transcripts. The results were similar to that of the first experiment in both quantitative and qualitative aspects. The first two principal components, $\mathrm{PC} 1$ and PC2, explained more than $50 \%$ of the variability, and a distinct response to septic plasma compared with uninfected plasma was observed (Figure 4A, left panel). However, there was significant heterogeneity observed among the samples stimulated with septic plasma.

Further analyses identified 2 parameters accounting for much of the observed heterogeneity among samples stimulated with septic plasma - sepsis severity and timing of sample collection. To stratify severity, patient samples were categorized into "severe" or "not severe" sepsis according to predefined criteria (see Methods). In contrast to patients with non-severe sepsis, patients with severe sepsis were markedly separated from uninfected controls. This result was confirmed by calculating the 

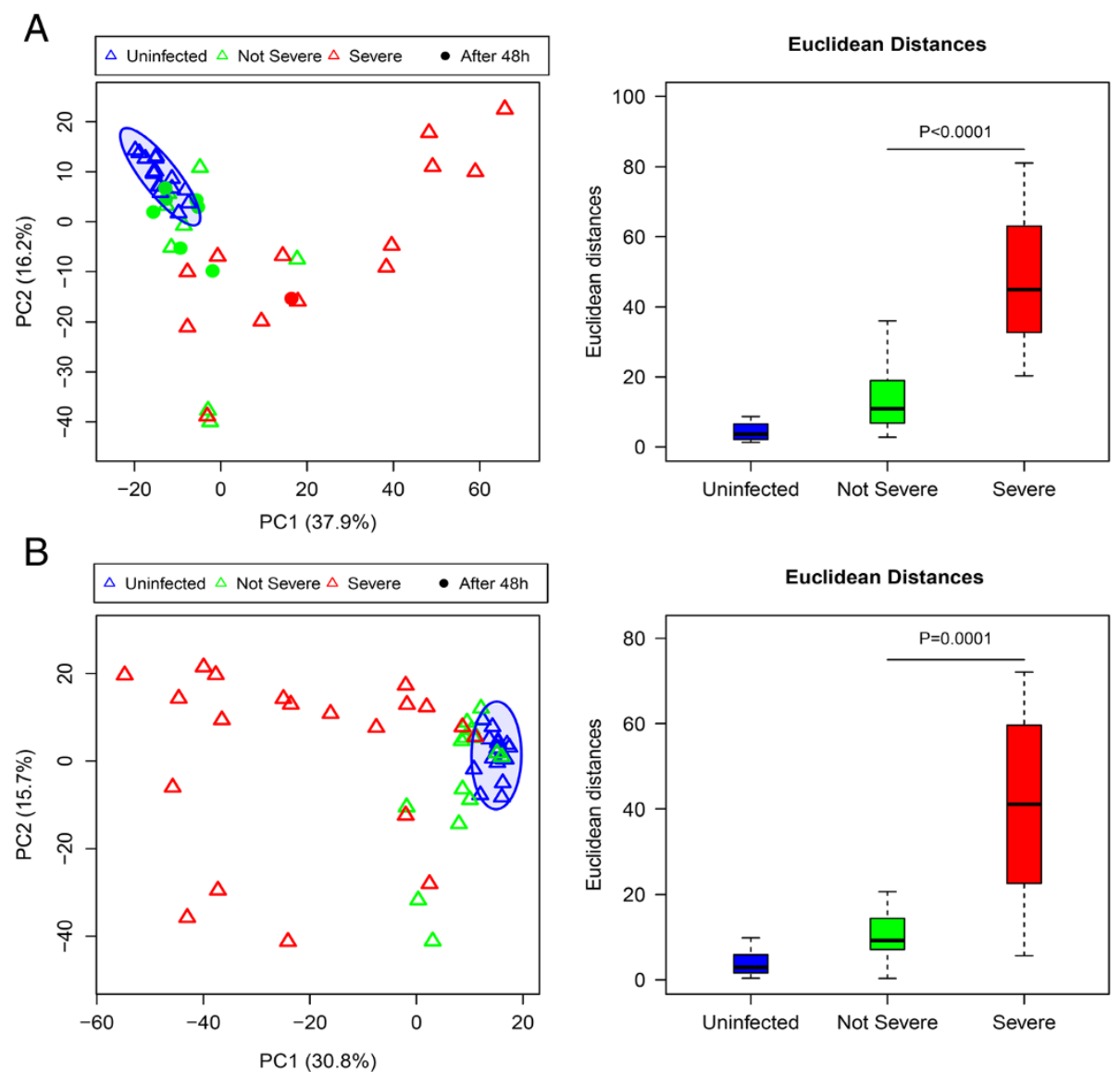

Figure 4 Responses to septic plasma measured in a PMN reporter assay for additional sets of subjects. Plasma samples from two additional sets of subjects were used in new sets of experiments: A and B corresponding to experiments II and III, respectively. PMNs used in both experiments were obtained from the same donor (donor $\mathrm{H} 2$ ) who had also participated in experiment I. Transcripts passing filter criteria of 2 fold-change and 100 difference in intensity (2FC100DIF) were used. PCA plots (left panels) were generated as described earlier. Colors and symbols indicate sample class and disease severity: red triangle, severe sepsis; green triangle, not severe sepsis; blue triangle, uninfected; and closed circle, plasma collected $>48 \mathrm{~h}$ after admission. Numbers in parenthesis indicate percentage of variance. Box plots (right panels) show Euclidean distances calculated for each sample from the center of ellipses corresponding to responses to plasma from uninfected controls. P-values were derived from a Mann-Whitney U-test.

Euclidean distance from the center of uninfected controls (ellipse in Figure 4A, right panel). The second important explanatory parameter identified was the timing of sample collection from hospital admission. There was no difference between transcriptional responses stimulated by septic plasma samples collected more than $48 \mathrm{~h}$ after admission and uninfected controls, whereas there was significant difference between septic plasma samples collected less than $48 \mathrm{~h}$ after admission and controls suggesting potential resolution of observed immunologic changes after treatment initiation. Numerous other demographic and clinical variables were investigated which did not appear to explain the observed heterogeneity (Additional file 9). The fact that most variability could be accounted for by sepsis severity suggests this is the primary factor determining the PMN response to plasma.

\section{Sepsis severity markers identified by the PMN reporter} system

Since disease severity impacted the magnitude of transcriptional response to septic plasma, we evaluated the potential value of our PMN reporter system for clinical applications. We conducted a third experiment using PMNs from the same donor as in a second experiment with an independent set of septic plasma samples all collected within $48 \mathrm{~h}$ of admission $(\mathrm{n}=35)$ and uninfected control samples $(n=19)$. PALO filtering returned 15,083 transcripts. The PCA plots generated from these transcripts showed similar results to that obtained in the second experiment confirming the marked separation of severe sepsis from not severe sepsis and controls and demonstrating sepsis severity the main variable affecting transcriptional responses (Figure 4B, Additional file 10). To assess variation of the cell donor source, we tested 
the same set of plasma samples with PMNs from an additional donor not used in either of the previous two experiments. PCA plots and Euclidean distance comparisons demonstrate similar findings as with the first donor suggesting this sort of PMN reporter system can be reproducible independent of the cell donor (Additional file 11).

We then used a RKNN-FS algorithm to identify markers for differentiating severe and not severe sepsis (Table 3). Data from the third experiment were used as the training set because it was the most balanced and largest dataset (severe sepsis $\mathrm{n}=20$; not severe sepsis $\mathrm{n}=15)$. RKNN-FS identified a set of 30 transcripts as being able to provide the highest accuracy $(82.02 \%)$ in predicting sample class in a leave one out cross-validation scheme. This biomarker signature reflects different amplitudes of PMN responses to septic plasma samples according to disease severity. Misclassification was observed only with some non-severe sepsis and uninfected controls. This result is due to the more similar transcriptional response between these two groups as seen in Figure 4. To illustrate the performance of the identified markers for predicting both severe and non-severe sepsis from uninfected controls by a ROC curve, we built prediction models by using SVM and RF algorithms. Data from experiment II (severe sepsis $\mathrm{n}=17$; not severe sepsis $n=12$ ) were used as a test set. The results demonstrated the high accuracy of the 30-transcript panel in predicting the response to plasma from severe sepsis samples; AUCs are 1 for both SVM and RF. The prediction accuracies of response to plasma from non-severe sepsis samples are slightly lower; the AUCs are 0.82 (95\% confidence interval, $0.78-1.00)$ and $0.90(95 \%$

Table 3 Sepsis severity markers identified by the PMN reporter system

\begin{tabular}{|c|c|c|c|c|}
\hline No. & Abbreviation & Accession number & Gene name & Illumina ID \\
\hline 1 & $A L G 10 B$ & GenBank:NM_001013620.3 & Asparagine-linked glycosylation 10 & ILMN_1730304 \\
\hline 2 & ARID5A & GenBank:NM_212481.1 & AT rich interactive domain $5 \mathrm{~A}$ & ILMN_1689700 \\
\hline 3 & $\mathrm{CCL} 22$ & GenBank:NM_002990.3 & Chemokine ( $\mathrm{C}-\mathrm{C}$ motif) ligand 22 & ILMN_2160476 \\
\hline 4 & CCND3 & GenBank:NM_001760.2 & Cyclin D3 & ILMN_1668721 \\
\hline 5 & $D M X L 2$ & GenBank:NM_015263.2 & Dmx-like 2 & ILMN_1705663 \\
\hline 6 & ECHDC3 & GenBank:NM_024693.2 & Enoyl Coenzyme A hydratase domain containing 3 & ILMN_2072178 \\
\hline 7 & EXOC5 & GenBank:NM_006544.3 & Exocyst complex component 5 & ILMN_1788625 \\
\hline 8 & FAM195A & GenBank:NM_138418.2 & Family with sequence similarity 195, member A & ILMN_1730523 \\
\hline 9 & FCGR2B & GenBank:XM_938851.1 & Fc fragment of IgG, low affinity $\| \mathrm{lb}$, receptor & ILMN_1804174 \\
\hline 10 & FKBP5 & GenBank:NM_004117.2 & FK506 binding protein 5 & ILMN_1778444 \\
\hline 11 & $\| 18 R 1$ & GenBank:NM_003855.2 & Interleukin 18 receptor 1 & ILMN_1781700 \\
\hline 12 & ILIR2 & GenBank:NM_004633.3 & Interleukin 1 receptor, type II & ILMN_1758371 \\
\hline 13 & KLF9 & GenBank:NM_001206.2 & Kruppel-like factor 9 & ILMN_1778523 \\
\hline 14 & MCOLN2 & GenBank:NM_153259.2 & Mucolipin 2 & ILMN_1660462 \\
\hline 15 & METTL6 & GenBank:NM_152396.2 & Methyltransferase like 6 & ILMN_1661998 \\
\hline 16 & MMP9 & GenBank:NM_004994.2 & Matrix metallopeptidase 9 & ILMN_1796316 \\
\hline 17 & NCRNA00120 & GenBank:NR_002767.1 & AKIRIN2 antisense RNA1 (non-protein coding) & ILMN_3239856 \\
\hline 18 & $P 2 R Y 2$ & GenBank:NM_176071.1 & Purinergic receptor $\mathrm{P} 2 \mathrm{Y}$ & ILMN_1723535 \\
\hline 19 & PCYOX1 & GenBank:NM_016297.2 & Prenylcysteine oxidase 1 & ILMN_2113535 \\
\hline 20 & PGM1 & GenBank:NM_002633.2 & Phosphoglucomutase 1 & ILMN_1800659 \\
\hline 21 & PIBF1 & GenBank:NM_006346.2 & Progesterone immunomodulatory binding factor 1 & ILMN_1758111 \\
\hline 22 & SEC24A & GenBank:NM_021982.1 & SEC24 family, member A & ILMN_2126832 \\
\hline 23 & SLC15A3 & GenBank:NM_016582.1 & Solute carrier family 15, member 3 & ILMN_2085862 \\
\hline 24 & $S L C 25 A 3$ & GenBank:NM_002635.2 & Solute carrier family 25, member 3 & ILMN_2332713 \\
\hline 25 & SMAP2 & GenBank:NM_022733.1 & Small ArfGAP2 & ILMN_1781468 \\
\hline 26 & TFRC & GenBank:NM_003234.1 & Transferrin receptor & ILMN_1674243 \\
\hline 27 & TLR2 & GenBank:NM_003264.3 & Toll-like receptor 2 & ILMN_1772387 \\
\hline 28 & TNFRSF9 & GenBank:NM_001561.4 & Tumor necrosis factor receptor superfamily, member 9 & ILMN_1813379 \\
\hline 29 & TPST1 & GenBank:NM_003596.2 & Tyrosylprotein sulfotransferase 1 & ILMN_1651950 \\
\hline 30 & YIPF5 & GenBank:NM_030799.6 & Yip1 domain family, member 5 & ILMN_1714756 \\
\hline
\end{tabular}


confidence interval, 0.66 - 0.99) for SVM and RF, respectively (Figure 5). Meaningful validation could not be done using data from experiment I because almost all of these plasma samples $(11 / 12)$ were obtained from patients with severe sepsis, 3 of which were collected at $>48 \mathrm{~h}$ after admission.

\section{Functional annotation of the candidate gene signature is a further indication of its relevance as a severity \\ biomarker}

Enrichment analyses were performed to characterize the functional relevance of our severity signature panel in GeneGo MetaCore. Collectively, these genes are involved in immune system response as their major biologic function (Figure 6A). Analyses for significant cellular process (Pathway Maps) also suggested their roles in cell cycle regulation (Figure $6 \mathrm{~B}$ ). The genes participating in these functions can be visualized on an interaction network (Figure 6C).

In addition to these enrichment analyses, many of our 30 classifier biomarkers have been previously shown to have potential roles in several dysregulated inflammatory conditions including sepsis and secondary organ dysfunction. Most of the 11 over-expressed transcripts have been independently identified as being of potential value as severity biomarkers in such inflammatory conditions. FKBP5 has been shown to contribute to the regulation of myeloid-derived suppressor cells (MDSCs) and changes in

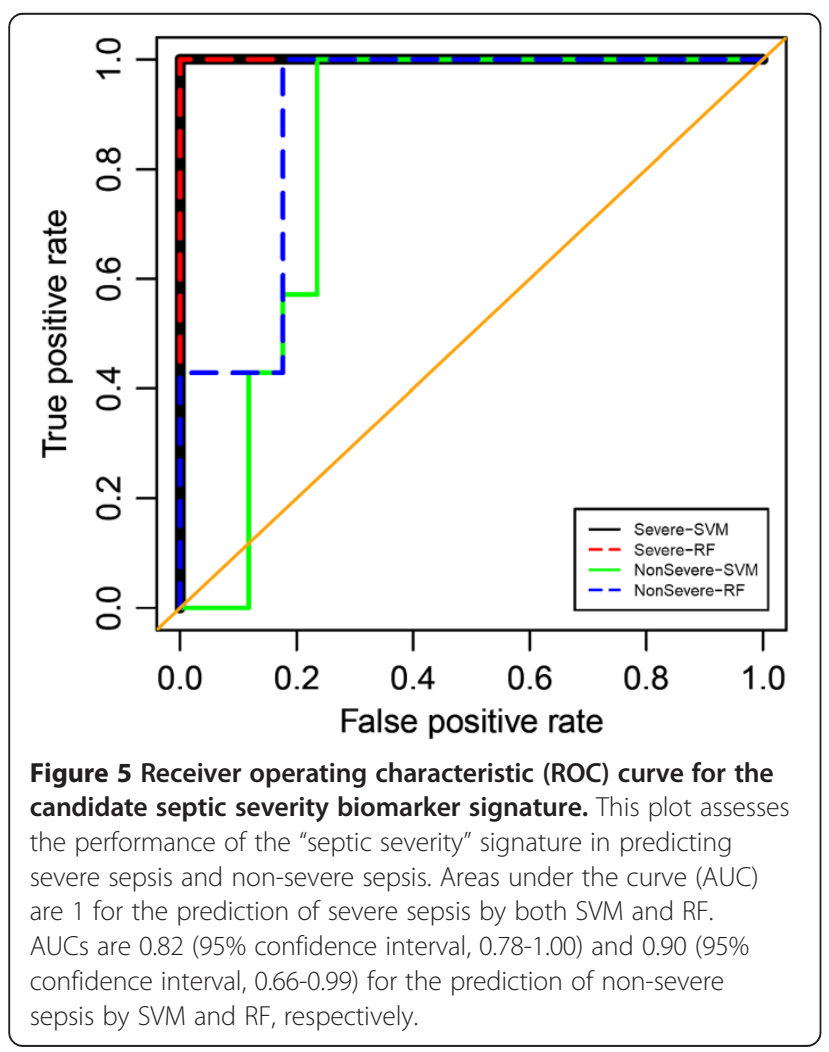

MDSCs have been shown important in a sepsis model [26,27]. TLR2 has been shown to be overexpressed in septic shock and also to play a role in acute kidney injury, which is an important consequence of severe sepsis and was part of our severity criteria [28,29]. TPST1 overexpression can be induced by lipopolysaccharides (LPS) and inhibition of TPST1 has been shown to affect macrophage signaling suggesting its role in the innate immune response [30]. ECHDC3 over-expression was reported in onset of acute coronary syndrome [31]. IL18R1 and IL1R2 belong to the IL1R family. IL18R1 plays a role in neutrophil migration and activation and has been identified as a biomarker for several systemic inflammatory conditions, such as surgery-induced inflammation and bacterial meningitis [32-34]. IL1R2 expression has been suggested to be a marker of sepsis and high circulating IL1R2 protein levels have been reported in critically-ill patients with sepsis $[35,36]$.

The functional relevance of the 19 under-expressed biomarkers was also investigated. MMP9 has been shown to have negative correlation with multiple organ dysfunction scores in sepsis [37]. FCGR2B deficient mice have been shown to have increased bacterial clearance and survival in sepsis [38]. TNFRSF9 is thought to be down-regulated by TNF- $\alpha$, and to enhance anti-apoptosis and subsequently induce inflammatory responses $[39,40]$. CCL22 is involved in regulating immune response by recruiting $\mathrm{T}$ helper cells and regulatory $\mathrm{T}$ cells and a sepsis model demonstrated CCL22 playing a role in enhancing neutrophil activation and chemotaxis [41-43]. P2RY2 has been shown to play a role in neutrophil chemotaxis in a mouse model and is also involved in the recruitment of PMNs to the lung resulting in acute lung injury in sepsis $[44,45]$.

Several other genes in our severity panel are involved in cell cycle and proliferation, for example, $K L F 9$, CCND3, YIPF5, ALG10B, and ARID5A. Taken together this literature shows well-established pathogenic roles in sepsis or other inflammatory conditions for much of our candidate biomarker panel and constitutes an external validation of our reporter assay system.

\section{Discussion}

This study reports the development and implementation of a "transcriptomic reporter assay" designed to investigate the immunogenicity of septic patient plasma and to our knowledge is the first published study employing immune cells in a whole genome transcriptomic reporter assay for an infectious process. One previous study investigated a focused transcriptional response of 1700 transcripts in cardiac myocytes cultured with septic serum [46]. Whole transcriptomic reporter assays have been employed successfully in previous studies of autoimmune diseases and have contributed to the understanding of disease pathogenesis in systemic onset juvenile idiopathic arthritis and 


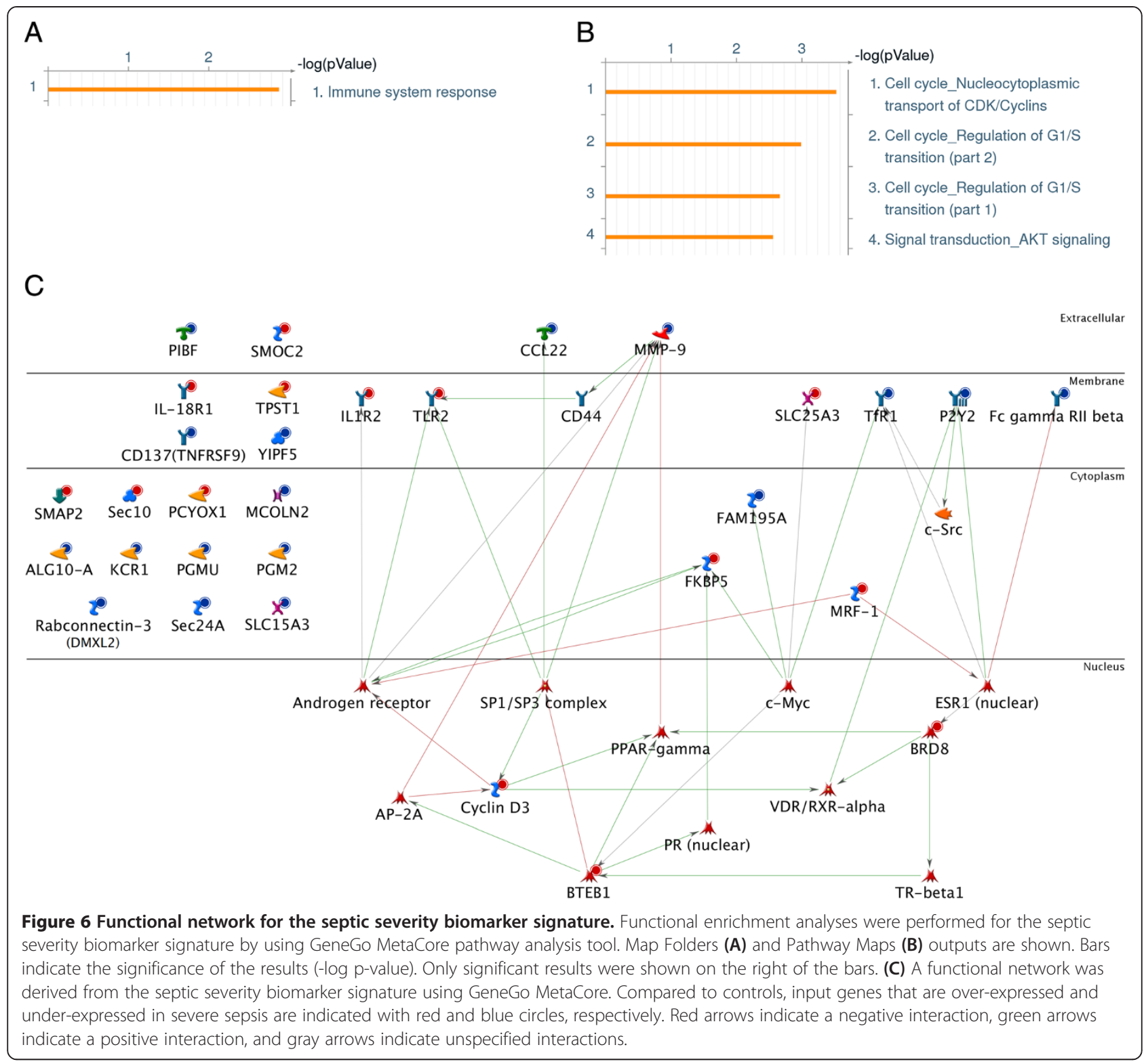

type 1 diabetes mellitus [7-11]. Our work helps further extend this technique into the infectious disease field and also confirms the utility of comparing performance of multiple leukocyte subsets.

Transcriptomic reporter assays such as this one are based on the fact that plasma carries diverse circulating immune mediators. Stimulation of immune cells by plasma can demonstrate the biological processes triggered by the immune responses of the host $[7,9]$. In the context of infection, a transcriptomic reporter assay may be detecting in part the responses triggered by exogenous molecules including pathogen associated molecular patterns (PAMPs) such as LPS, lipoproteins, and peptidoglycans [47]. However such an assay also reflects the responses triggered by diverse endogenous signaling molecules including cytokines and damage associated molecular patterns (DAMPs) among others [48].

Our work identified that for investigation of sepsis, PMNs served as the best reporter cells in a side-by-side comparison with PBMCs and MoDCs. PMNs were better sensors of immunostimulatory factors present in plasma, displaying improved ability to discriminate septic from uninfected subjects, and PMNs mobilized the most robust immune transcriptional program. This finding was not initially expected given that PBMCs have been to date the preferred "serum sensing" cell reporter system [9], and given that dendritic cells are well known for their sentinel role in the immune system and have ability to respond to a wide range of immune triggers. However our findings are consistent with the role of 
PMNs, which serve as the first line of the cellular innate immune response and are a major source of acute phase immune mediators $[49,50]$. Further our results support previous work investigating the robust transcriptional response of PMN in sepsis. For example, Wong et al. demonstrated from leukocyte transcriptional profiling in pediatric septic shock that the number of differentially expressed genes in PMNs was greater than in monocytes or lymphocytes suggesting repression of adaptive immunity gene programs in early sepsis [51]. De Kleijn et al. demonstrated a robust set of functional gene networksdifferentially expressed by PMNs after both in vivo and ex vivo exposure to LPS that related to extended survival and the regulation of inflammatory responses [52]. It should be noted that the arrays of receptors expressed by different immune cell types vary widely, and that while our report indicate that neutrophils are especially well equipped to respond to plasma from septic patients it may not be the most appropriate cell type in other settings.

The PMN cell reporter system coupled with whole transcriptome readout allowed identification of a severity signature for sepsis that was highly accurate in two independent datasets. Current guidelines for diagnosing sepsis and grading severity are based on multiple clinical parameters, which can be inaccurate and do not predict prognosis well [15]. Although many potential biomarkers including cytokines, coagulation factors, and several others have been investigated for sepsis diagnosis and prognosis, none have proved reliable enough to enter routine clinical practice and so there is a need for better markers for risk stratification of septic patients to guide treatment and prognosis [17-19]. Elucidating the biologic mechanisms that differ among sepsis patients will help advance this field [12,20]. The promising performance of the PMN transcriptomic reporter assay presented here to stratify patients with sepsis by severity offers a novel and attractive platform for the development of biomarker signatures in sepsis. Similarly, the ability to select a gene panel that was specific to stimulation with plasma from severe sepsis patients shows the utility of this method to better understand the immunopathogenesis of sepsis.

Additional investigation is warranted by these results. The use of three independent cohorts of patients demonstrates reproducibility, but investigation with profiling of longitudinal samples will be necessary to further validate our severity assessment and to determine its potential value in monitoring disease progression and the response to treatment. Seeing consistent results using three separate PMN donors suggests our results can be reproducible independent of donor source, however further studies are necessary to determine how much variability donor source could introduce. Similarly extending our study to stable cell lines such as the neutrophil-like
HL-60 line could be useful to develop a standardized assay, an approach that has been previously investigated in the context of type 1 diabetes research [53]. Perhaps most important, comparison of transcriptional profiles elicited from similar clinical conditions due to sterile inflammation (e.g. non-infectious SIRS or major surgery) is essential to determine the specificity of the severity signature described in this study. Also further work towards using this approach to identify a causative pathogen could hold potential - in our case the relatively small sample size and diversity of pathogens made it difficult to address this issue.

While at present a transcriptomic PMN reporter assay is not ideal for applications at the bedside given challenges for standardization of cell lines and data processing, technological advances in automation of sample processing and availability of polymerase chain reaction (PCR)-based amplification would make the implementation of a similar but more targeted assay feasible [54,55]. This approach could also serve as a novel platform for biomarker discovery and the development of novel clinical tests that could improve diagnosis and prognosis in sepsis. Moreover, this type of neutrophil transcriptomic reporter assay is likely to prove valuable for the investigation of other immunologically mediated diseases.

\section{Conclusions}

We demonstrated the utility and accuracy of a neutrophil reporter assay coupled with whole transcriptome readout for predicting sepsis severity. We also demonstrated that this technique identifies important functional networks involved in the pathogenesis of sepsis.

\section{Additional files}

Additional file 1: Table S1. Demographic and clinical data for subjects used in experiment 1 .

Additional file 2: Table S2. Demographic and clinical data for subjects used in experiment II.

Additional file 3: Table S3. Demographic and clinical data for subjects used in experiment III.

Additional file 4: Figure S1. Box plot showing Euclidean distances from the PCA plots on Figure 2. Euclidian distances were calculated for each sample from the center of the ellipses corresponding to responses to plasma from uninfected controls in each reporter system (See Figure 2). Reporter cells and types of plasma are indicated on the $\mathrm{x}$-axis. P-values were derived from a Mann-Whitney U-test.

Additional file 5: Figure S2. Principal component analyses of transcriptional responses to septic plasma in three different reporter cells systems. A subset of septic plasma samples eliciting robust transcriptional responses consistently across all three cell reporter systems is indicated with red triangles on these PCA plots derived from Figure 2. Color indicates study groups (blue = uninfected plasma; red= septic plasma). An ellipsis indicates 95\% confidence interval of data from the corresponding group (indicated by color). Number in parenthesis indicates percentage of variance. See the legend for Figure 2 for more details. 
Additional file 6: Expression profile of Transcripts changing by at least 2-fold compared to their own unstimulated controls in each reporter system.

Additional file 7: Figure S3. Summary of transcripts expressed in each reporter cell system. Venn diagram demonstrating overlap of the 1,366 differentially genes (from Figure 3 and Additional file 6) for the 3 reporter cell types.

Additional file 8: Predicted transcription factors from genes overexpressed in each reporter cell system.

Additional file 9: Figure S4. Transcriptional responses and additional clinical data association (Experiment II). PCA plot from experiment II (Figure 4A) overlaid with additional clinical information for the sepsis patients: type of bacterial infection, Gram stain of bacterial infection, age (divided as $<60$ and $\geq 60$ years-old), gender, presence of underlying diabetes mellitus, and presence of underlying chronic kidney disease.

Additional file 10: Figure S5. Transcriptional responses and additional clinical data association (Experiment III). PCA plot from experiment III (Figure 4B) overlaid with additional clinical information for the sepsis patients: type of bacterial infection, Gram stain of bacterial infection, age (divided as $<60$ and $\geq 60$ years-old), gender, presence of underlying diabetes mellitus, and presence of underlying chronic kidney disease.

Additional file 11: Figure S6. Responses to septic plasma measured in a PMN reporter assay using PMNs from an additional healthy donor. Results from experiment III as shown in Figure $4 \mathrm{~B}$ are replicated here in (A). PMNs from an additional donor were treated with the same set of plasma samples from experiment III (B) demonstrating similar responses. See Figure 4 legend for further details.

\section{Abbreviations}

AUC: Area under the curve; DAMP: Damage-associated molecular pattern; KNN: K-nearest neighbor; LPS: Lipopolysaccharides; MODC: Monocyte-derived dendritic cell; NCBI: National Center for Biotechnology Information; PALO: Presence in at least one sample: PAMP: Pathogen-associated molecular pattern; PBMC: Peripheral blood mononuclear cell; PC: Principal component; PCA: Principal component analysis; PCR: Polymerase chain reaction; PMN: Polymorphonuclear cell; RF: Random forest; RKNN-FS: Random k-nearest neighbor - feature selection; ROC: Receiver operating characteristic; SIRS: Systemic inflammatory response syndrome; SVM: Support vector machine.

\section{Competing interests}

The authors declare that they have no competing interest.

\section{Authors' contributions}

$\mathrm{DC}$ and $\mathrm{GL}$ designed and conceived research; DR, DS, SB, CK, KO, and QAN performed experiments. WS provided specimens and clinical data. PK, EW, MCA, SP, and MM analyzed data. VG contributed new reagents/analytic tools. PK, DR, LC, MCA, PL, GL, and DC wrote the paper. All authors read and approved the final manuscript.

\section{Acknowledgements}

We thank all patients and volunteers who participated in the study, the staff of Khon Kaen Regional Hospital for patient recruitment, Brenda Norris for reviewing and submitting the manuscript, and all members of the Systems Immunology Division, BRI for helpful discussions. This work was supported by the National Institutes of Health (U01 Grant no. U01Al082110 to DC).

\section{Author details}

'Systems Immunology Division, Benaroya Research Institute, 1201 Ninth Avenue, Seattle, WA 98101, USA. ${ }^{2}$ Centre for Research and Development of Medical Diagnostic Laboratories (CMDL), Faculty of Associated Medical Sciences, Khon Kaen University, 123 Friendship Highway, Muang, Khon Kaen 40002, Thailand. ${ }^{3}$ Department of Internal Medicine, Hospital La Conception, 147 Blvd Baille, Marseille 13005, France. ${ }^{4}$ Department of Internal Medicine, Khon Kaen Regional Hospital, 54 Srichan Road, Muang, Khon Kaen 40000, Thailand.

\section{References}

1. Germain RN, Meier-Schellersheim M, Nita-Lazar A, Fraser ID: Systems biology in immunology: a computational modeling perspective. Annu Rev Immunol 2011, 29:527-585.

2. Nakaya HI, Li S, Pulendran B: Systems vaccinology: learning to compute the behavior of vaccine induced immunity. Wiley Interdiscip Rev Syst Biol Med 2012, 4:193-205.

3. Pankla R, Buddhisa S, Berry M, Blankenship DM, Bancroft GJ, Banchereau J, Lertmemongkolchai G, Chaussabel D: Genomic transcriptional profiling identifies a candidate blood biomarker signature for the diagnosis of septicemic melioidosis. Genome Biol 2009, 10:R127.

4. Berry MP, Graham CM, McNab FW, Xu Z, Bloch SA, Oni T, Wilkinson KA Banchereau R, Skinner J, Wilkinson RJ, Quinn C, Blankenship D, Dhawan R, Cush JJ, Mejias A, Ramilo O, Kon OM, Pascual V, Banchereau J, Chaussabel D, O'Garra A: An interferon-inducible neutrophil-driven blood transcriptional signature in human tuberculosis. Nature 2010, 466:973-977.

5. Chaussabel D, Pascual V, Banchereau J: Assessing the human immune system through blood transcriptomics. BMC Biol 2010, 8:84.

6. Paul D, Kumar A, Gajbhiye A, Santra MK, Srikanth R: Mass spectrometrybased proteomics in molecular diagnostics: discovery of cancer biomarkers using tissue culture. Biomed Res Int 2013, $2013: 783131$.

7. Pascual V, Allantaz F, Arce E, Punaro M, Banchereau J: Role of interleukin-1 (IL-1) in the pathogenesis of systemic onset juvenile idiopathic arthritis and clinical response to IL-1 blockade. J Exp Med 2005, 201:1479-1486.

8. Quartier P, Allantaz F, Cimaz R, Pillet P, Messiaen C, Bardin C, Bossuyt X Boutten A, Bienvenu J, Duquesne A, Richer O, Chaussabel D, Mogenet A, Banchereau J, Treluyer JM, Landais P, Pascual V: A multicentre, randomised, double-blind, placebo-controlled trial with the interleukin-1 receptor antagonist anakinra in patients with systemic-onset juvenile idiopathic arthritis (ANAJIS trial). Ann Rheum Dis 2011, 70:747-754.

9. Wang X, Jia S, Geoffrey R, Alemzadeh R, Ghosh S, Hessner MJ: Identification of a molecular signature in human type 1 diabetes mellitus using serum and functional genomics. J Immunol 2008, 180:1929-1937.

10. Jackson A, McWilliams C, Kaizer E, Chaussabel D, Glaser C, Noguchi H, Matsumoto S, Levy MF, Naziruddin B: Gene expression profiling of human pancreatic islets undergoing a simulated process of instant bloodmediated inflammatory reaction. Transplant Proc 2008, 40:430-432.

11. Jackson AM, Kanak MA, Grishman EK, Chaussabel D, Levy MF, Naziruddin B: Gene expression changes in human islets exposed to type 1 diabetic serum. Islets 2012, 4:312-319.

12. Skrupky LP, Kerby PW, Hotchkiss RS: Advances in the management of sepsis and the understanding of key immunologic defects. Anesthesiology 2011, 115:1349-1362.

13. Rivers E, Nguyen B, Havstad S, Ressler J, Muzzin A, Knoblich B, Peterson E, Tomlanovich M: Early goal-directed therapy in the treatment of severe sepsis and septic shock. N Engl J Med 2001, 345:1368-1377.

14. Kumar A, Roberts D, Wood KE, Light B, Parrillo JE, Sharma S, Suppes R, Feinstein D, Zanotti S, Taiberg L, Gurka D, Cheang M: Duration of hypotension before initiation of effective antimicrobial therapy is the critical determinant of survival in human septic shock. Crit Care Med 2006, 34:1589-1596.

15. Dellinger RP, Levy MM, Rhodes A, Annane D, Gerlach H, Opal SM, Sevransky JE, Sprung CL, Douglas IS, Jaeschke R, Osborn TM, Nunnally ME, Townsend SR, Reinhart K, Kleinpell RM, Angus DC, Deutschman CS, Machado FR, Rubenfeld GD, Webb S, Beale RJ, Vincent JL, Moreno R: Surviving Sepsis Campaign: international guidelines for management of severe sepsis and septic shock, 2012. Intensive Care Med 2013, 39:165-228.

16. Angus DC, van der Poll T: Severe sepsis and septic shock. N Engl J Med 2013, 369:840-851

17. Faix JD: Biomarkers of sepsis. Crit Rev Clin Lab Sci 2013, 50:23-36

18. Schuetz P, Albrich W, Mueller B: Procalcitonin for diagnosis of infection and guide to antibiotic decisions: past, present and future. BMC Med 2011, 9:107.

19. Gibot S, Bene MC, Noel R, Massin F, Guy J, Cravoisy A, Barraud D, De Carvalho Bittencourt M, Quenot JP, Bollaert PE, Faure G, Charles PE: Combination biomarkers to diagnose sepsis in the critically ill patient. Am J Respir Crit Care Med 2012, 186:65-71.

20. Lichtenstern C, Brenner T, Bardenheuer HJ, Weigand MA: Predictors of survival in sepsis: what is the best inflammatory marker to measure? Curr Opin Infect Dis 2012, 25:328-336.

21. Chanchamroen S, Kewcharoenwong C, Susaengrat W, Ato M, Lertmemongkolchai G: Human polymorphonuclear neutrophil responses 
to Burkholderia pseudomallei in healthy and diabetic subjects. Infect Immun 2009, 77:456-463.

22. Rinchai $D$, Khaenam P, Kewcharoenwong C, Buddhisa S, Pankla R, Chaussabel D, Bancroft GJ, Lertmemongkolchai G: Production of interleukin-27 by human neutrophils regulates their function during bacterial infection. Eur J Immunol 2012, 42:3280-3290.

23. Tippayawat $P$, Pinsiri M, Rinchai D, Riyapa D, Romphruk A, Gan YH, Houghton RL, Felgner PL, Titball RW, Stevens MP, Galyov EE, Bancroft GJ, Lertmemongkolchai G: Burkholderia pseudomallei proteins presented by monocyte-derived dendritic cells stimulate human memory $T$ cells in vitro. Infect Immun 2011, 79:305-313.

24. Obermoser G, Presnell S, Domico K, Xu H, Wang Y, Anguiano E, ThompsonSnipes L, Ranganathan R, Zeitner B, Bjork A, Anderson D, Speake C, Ruchaud E, Skinner J, Alsina L, Sharma M, Dutartre H, Cepika A, Israelsson E, Nguyen P, Nguyen QA, Harrod AC, Zurawski SM, Pascual V, Ueno H, Nepom GT, Quinn C, Blankenship D, Palucka K, Banchereau J, et al: Systems scale interactive exploration reveals quantitative and qualitative differences in response to influenza and pneumococcal vaccines. Immunity 2013, 38:831-844.

25. Li S, Harner EJ, Adjeroh DA: Random KNN feature selection - a fast and stable alternative to Random Forests. BMC Bioinformatics 2011, 12:450.

26. Brudecki L, Ferguson DA, McCall CE, El Gazzar M: Myeloid-derived suppressor cells evolve during sepsis and can enhance or attenuate the systemic inflammatory response. Infect Immun 2012, 80:2026-2034.

27. Kim YS, Kim YJ, Lee JM, Kim EK, Park YJ, Choe SK, Ko HJ, Kang CY: Functional changes in myeloid-derived suppressor cells (MDSCs) during tumor growth: FKBP51 contributes to the regulation of the immunosuppressive function of MDSCs. J Immunol 2012, 188:4226-4234.

28. Castoldi A, Braga TT, Correa-Costa M, Aguiar CF, Bassi EJ, Correa-Silva R, Elias RM, Salvador F, Moraes-Vieira PM, Cenedeze MA, Reis MA, Hiyane MI, Pacheco-Silva A, Goncalves GM, Saraiva Camara NO: TLR2, TLR4 and the MYD88 signaling pathway are crucial for neutrophil migration in acute kidney injury induced by sepsis. PLoS One 2012, 7:e37584.

29. Ubagai T, Tansho S, leki R, Ono Y: Evaluation of TREM1 gene expression in circulating polymorphonuclear leukocytes and its inverse correlation with the severity of pathophysiological conditions in patients with acute bacterial infections. Jpn J Infect Dis 2012, 65:376-382.

30. Yang IV, Jiang W, Rutledge HR, Lackford B, Warg LA, De Arras L, Alper $S$, Schwartz DA, Pisetsky DS: Identification of novel innate immune genes by transcriptional profiling of macrophages stimulated with TLR ligands. Mol Immunol 2011, 48:1886-1895.

31. Silbiger VN, Luchessi AD, Hirata RD, Lima-Neto LG, Cavichioli D, Carracedo A, Brion M, Dopazo J, Garcia-Garcia F, Dos Santos ES, Ramos RF, Sampaio MF, Armaganijan D, Sousa AG, Hirata MH: Novel genes detected by transcriptional profiling from whole-blood cells in patients with early onset of acute coronary syndrome. Clin Chim Acta 2013, 421C:184-190.

32. Lill M, Koks S, Soomets U, Schalkwyk LC, Fernandes C, Lutsar I, Taba P. Peripheral blood RNA gene expression profiling in patients with bacterial meningitis. Front Neurosci 2013, 7:33.

33. Leung BP, Culshaw S, Gracie JA, Hunter D, Canetti CA, Campbell C, Cunha F, Liew FY, McInnes IB: A role for IL-18 in neutrophil activation. J Immunol 2001, 167:2879-2886.

34. Buvanendran A, Mitchell K, Kroin JS, ladarola MJ: Cytokine gene expression after total hip arthroplasty: surgical site versus circulating neutrophil response. Anesth Analg 2009, 109:959-964.

35. Muller B, Peri G, Doni A, Perruchoud AP, Landmann R, Pasqualini F, Mantovani A: High circulating levels of the IL-1 type II decoy receptor in critically ill patients with sepsis: association of high decoy receptor levels with glucocorticoid administration. J Leukoc Biol 2002, 72:643-649.

36. Chinnaiyan AM, Huber-Lang M, Kumar-Sinha C, Barrette TR, Shankar-Sinha S, Sarma VJ, Padgaonkar VA, Ward PA: Molecular signatures of sepsis: multiorgan gene expression profiles of systemic inflammation. Am J Pathol 2001, 159:1199-1209.

37. Yazdan-Ashoori P, Liaw P, Toltl L, Webb B, Kilmer G, Carter DE, Fraser DD Elevated plasma matrix metalloproteinases and their tissue inhibitors in patients with severe sepsis. J Crit Care 2011, 26:556-565.

38. Clatworthy MR, Smith KG: FcgammaRllb balances efficient pathogen clearance and the cytokine-mediated consequences of sepsis. J Exp Med 2004, 199:717-723.
39. Heinisch IV, Daigle I, Knopfli B, Simon HU: CD137 activation abrogates granulocyte-macrophage colony-stimulating factor-mediated anti-apoptosis in neutrophils. Eur J Immunol 2000, 30:3441-3446.

40. Nguyen QT, Nguyen TH, Ju SA, Lee YS, Han SH, Lee SC, Kwon BS, Yu R, Kim GY, Lee BJ, Kim BS: CD137 on neutrophils plays dual roles in anti-bacterial responses against Gram-positive and Gram-negative bacteria infection. Infect Immun 2013, 81:2168-2177.

41. Nishikawa $\mathrm{H}$, Sakaguchi S: Regulatory T cells in tumor immunity. Int J Cancer 2010, 127:759-767.

42. Shimada $Y$, Takehara $K$, Sato S: Both Th2 and Th1 chemokines (TARC/ CCL17, MDC/CCL22, and Mig/CXCL9) are elevated in sera from patients with atopic dermatitis. J Dermatol Sci 2004, 34:201-208.

43. Kornerup KN, Salmon GP, Pitchford SC, Liu WL, Page CP: Circulating platelet-neutrophil complexes are important for subsequent neutrophil activation and migration. J Appl Physiol 2010, 109:758-767.

44. Inoue $Y$, Chen Y, Hirsh MI, Yip L, Junger WG: A3 and P2Y2 receptors control the recruitment of neutrophils to the lungs in a mouse model of sepsis. Shock 2008, 30:173-177.

45. Chen $Y$, Corriden R, Inoue Y, Yip L, Hashiguchi N, Zinkernagel A, Nizet $\mathrm{V}$, Insel PA, Junger WG: ATP release guides neutrophil chemotaxis via P2Y2 and A3 receptors. Science 2006, 314:1792-1795.

46. Hussein S, Michael P, Brabant D, Omri A, Narain R, Passi K, Ramana CV, Parrillo JE, Kumar A, Parissenti A, Kumar A: Characterization of human septic sera induced gene expression modulation in human myocytes. Int J Clin Exp Med 2009, 2:131-148.

47. Mogensen TH: Pathogen recognition and inflammatory signaling in innate immune defenses. Clin Microbiol Rev 2009, 22:240-273.

48. Zhang Q, Raoof M, Chen Y, Sumi Y, Sursal T, Junger W, Brohi K, Itagaki K, Hauser CJ: Circulating mitochondrial DAMPs cause inflammatory responses to injury. Nature 2010, 464:104-107.

49. Kolaczkowska E, Kubes P: Neutrophil recruitment and function in health and inflammation. Nat Rev Immunol 2013, 13:159-175.

50. Mantovani A, Cassatella MA, Costantini C, Jaillon S: Neutrophils in the activation and regulation of innate and adaptive immunity. Nat Rev Immunol 2011, 11:519-531.

51. Wong HR, Freishtat RJ, Monaco M, Odoms K, Shanley TP: Leukocyte subsetderived genome-wide expression profiles in pediatric septic shock. Pediatr Crit Care Med 2010, 11:349-355.

52. De Kleijn S, Kox M, Sama IE, Pillay J, Van Diepen A, Huijnen MA, van der Hoeven JG, Ferwerda G, Hermans PW, Pickkers P: Transcriptome kinetics of circulating neutrophils during human experimental endotoxemia. PLoS One 2012, 7:e38255.

53. Jia S, Kaldunski M, Jailwala P, Geoffrey R, Kramer J, Wang X, Hessner MJ: Use of transcriptional signatures induced in lymphoid and myeloid cell lines as an inflammatory biomarker in Type 1 diabetes. Physiol Genomics 2011, 43:697-709.

54. Kulinsky L, Noroozi Z, Madou M: Present technology and future trends in point-of-care microfluidic diagnostics. Methods Mol Biol 2013, 949:3-23.

55. Xu M, Qin X, Astion ML, Rutledge JC, Simpson J, Jerome KR, Englund JA, Zerr DM, Migita RT, Rich S, Childs JC, Cent A, Del Beccaro MA: Implementation of filmarray respiratory viral panel in a core laboratory improves testing turnaround time and patient care. Am J Clin Pathol 2013, 139:118-123.

\section{doi:10.1186/1479-5876-12-65}

Cite this article as: Khaenam et al: A transcriptomic reporter assay employing neutrophils to measure immunogenic activity of septic patients' plasma. Journal of Translational Medicine 2014 12:65. 